\title{
GRID SENSITIVITY AND AERODYNAMIC OPTIMIZATION OF GENERIC AIRFOILS *
}

\author{
Ideen Sadrehaghighi ${ }^{\dagger}$ \\ Old Dominion University, Norfolk, Virginia 23529-0247 \\ Robert E. Smith ${ }^{\ddagger}$ \\ NASA Langley Research Center, Hampton, Virginia 23681-0001 \\ Surendra N. Tiwari ${ }^{\S}$ \\ Old Dominion University, Norfolk, Virginia 23529-0247
}

\begin{abstract}
An algorithm is developed to obtain the grid sensitivity with respect to design parameters for aerodynamic optimization. The procedure is advocating a novel (geometrical) parameterization using spline functions such as NURBS (Non-Uniform Rational BSplines) for defining the airfoil geometry. An interactive algebraic grid generation technique is employed to generate C-type grids around airfoils. The grid sensitivity of the domain with respect to geometric design parameters has been obtained by direct differentiation of the grid equations. A hybrid approach is proposed for more geometrically complex configurations such as a wing or fuselage. The aerodynamic sensitivity coefficients are obtained by direct differentiation of the compressible two-dimensional thinlayer Navier-Stokes equations. An optimization package has been introduced into the algorithm in order to optimize the airfoil surface. Results demonstrate a substantially improved design due to maximized lift/drag ratio of the airfoil.
\end{abstract}

\section{INTRODUCTION}

An essential element in design and optimization of aerodynamic surfaces is acquiring the sensitivity of aerodynamic surface forces with respect to design parameters. ${ }^{1-3}$ Several methods concerning the derivation of sensitivity equations are currently available. Among the most frequently mentioned are

\footnotetext{
* Copyright (C) 1995 by the American Institute of Aeronautics and Astronautics, Inc. All rights reserved.

${ }^{\dagger}$ Research Associate, Department of Mechanical Engineering, AIAA member.

$\ddagger$ Senior Research Engineer, Associate Fellow, AIAA.

$\S$ Eminent Professor, Department of Mechanical Engineering, Associate Fellow, AIAA.
}

Direct Differentiation (DD), Adjoint Variable (AV), Symbolic Differentiation (SD), Automatic Differentiation (AD), and Finite Difference (FD). Each technique has its own unique characteristics. The Direct Differentiation, adopted in this study, has the advantage of being exact, due to direct differentiation of governing equations with respect to design parameters. There are two basic components in obtaining aerodynamic sensitivity. They are: (1) obtaining the sensitivity of the governing equations with respect to the state variables, and (2) obtaining the sensitivity of the grid with respect to the design parameters. The sensitivity of the state variables with respect to the design parameters are described by a set of linear-algebraic relation. These systems of equations can be solved directly by a LU decomposition of the coefficient matrix. This direct inversion procedure becomes extremely expensive as the problem dimension increases. A hybrid approach of an efficient banded matrix solver with influence of off-diagonal elements iterated can be implemented to overcome this difficulty. ${ }^{2}$

After reviewing relevant literature, it is apparent that one aspect of aerodynamic sensitivity analysis, namely grid sensitivity, has not been investigated extensively. The grid sensitivity algorithms in most of these studies are based on structural design models. Such models, although sufficient for preliminary or conceptional design, are not acceptable for detailed design analysis. Careless grid sensitivity evaluations, would introduce gradient errors within the sensitivity module, therefore, infecting the overall optimization process. Development of an efficient and reliable grid sensitivity module with special emphasis on aerodynamic applications appears essential.

Among two major classes of grid generation systems (Algebraic, Differential), algebraic grid generation systems are ideally suited for achieving this ob- 
jective. The explicit formulation, resulting in a fast and suitable grid, enables direct differentiation of grid coordinates with respect to design parameters. ${ }^{4,5}$ The underlying effort here is to avoid the time consuming and costly numerical differentiation. In addition, the analytical derivatives are exact, a desirable feature for sensitivity analysis. An important ingredient of grid sensitivity is the surface parameterization. The most general parameterization would be to specify every grid point on the surface as a design parameter. This, although convenient, is unacceptable due to high computational cost. It is essential to keep the number of parameters as low as possible to avoid a surge on computational expenses. An analytical parameterization, may alleviate that problem but it suffers from lack of generality. A compromise would be using spline functions such as a Bezier or B-Spline function to represent the surface. In this manner, most aerodynamically inclined surfaces can be represented with only a few control (design) parameters.

\section{SURFACE MODELING AND GRID GENERATION}

Among many ideas proposed for generating any arbitrary surface, the approximative techniques of using spline functions are gaining a wide range of popularity. The most commonly used approximative representation is the Non-Uniform Rational BSpline (NURBS) function. They provide a powerful geometric tool for representing both analytic shapes (conics, quadrics, surfaces of revolution, etc.) and free-form surfaces. ${ }^{6}$ The surface is influenced by a set of control points and weights where unlike interpolating schemes the control points might not be at the surface itself. By changing the control points and corresponding weights, the designer can influence the surface with a great degree of flexibility without compromising the accuracy of the design. The relation for a NURBS curve is

$$
\begin{gathered}
\mathbf{X}(r)=\sum_{i=0}^{n} R_{i, p}(r) \mathbf{D}_{i} \quad i=0, \ldots, n \\
R_{i, p}(r)=\frac{N_{i, p}(r) \omega_{i}}{\sum_{i=0}^{n} N_{i, p}(r) \omega_{i}}
\end{gathered}
$$

where $\mathbf{X}(r)$ is the vector valued surface coordinate in the $r$-direction, $\mathbf{D}_{i}$ are the control points (forming a control polygon), $\omega_{i}$ are weights, $N_{i, p}(r)$ are the $p$-th degree B-Spline basis function, and $R_{i, p}(r)$ are known as the Rational basis functions.

Figure 1 illustrates a seven control point representation of a generic airfoil. The points at the leading

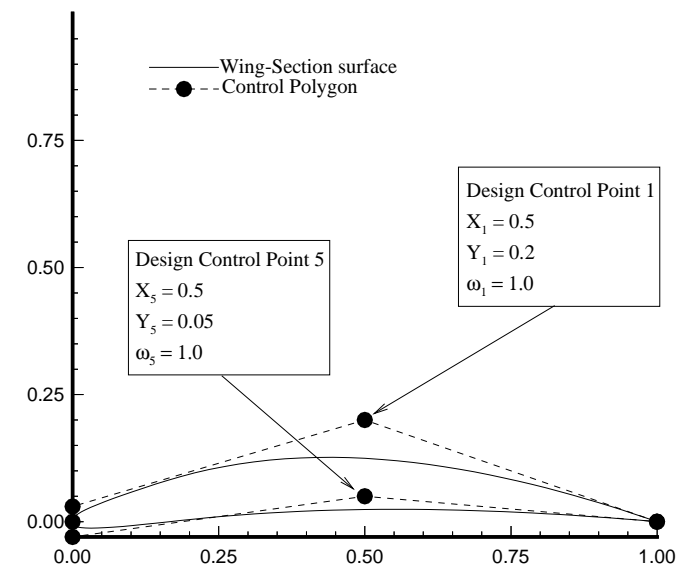

Figure 1: Seven control point representation of a generic airfoil

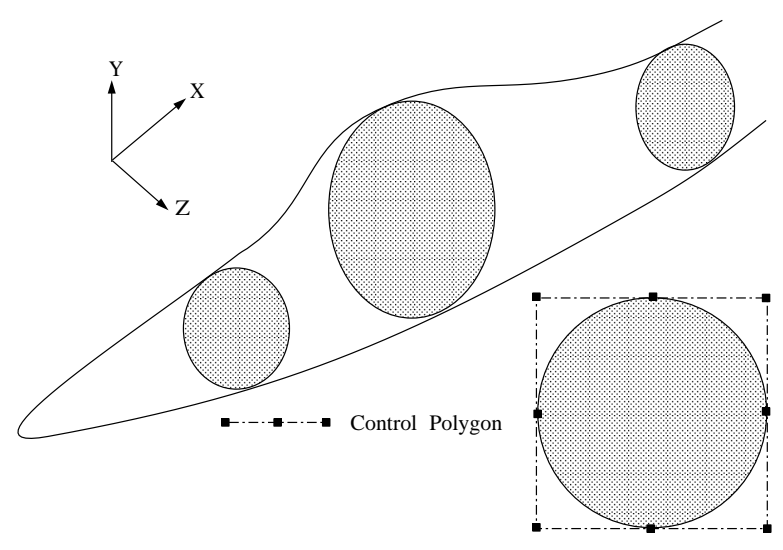

Figure 2: Critical fuselage cross-sections

and trailing edges are fixed. Two control points at the $0 \%$ chord are used to affect the bluntness of the section. Similar procedure can be applied to other airfoil geometries such as NACA four or five digit series. The choice for number of control points and their locations are best determined using an inverse B-Spline interpolation of the initial data. ${ }^{6}$ The algorithm yields a system of linear equations with a positive and banded coefficient matrix. Therefore, it can be solved safely using techniques such as Gaussian elemination without pivoting. The procedure can be easily extended to cross-sectional configurations, when critical cross-sections are defined by several circular conic sections, and the intermediate surfaces have been generated using linear interpolation as shown in Fig. 2. Increasing the weights would deform the circular segments to other conic segments 


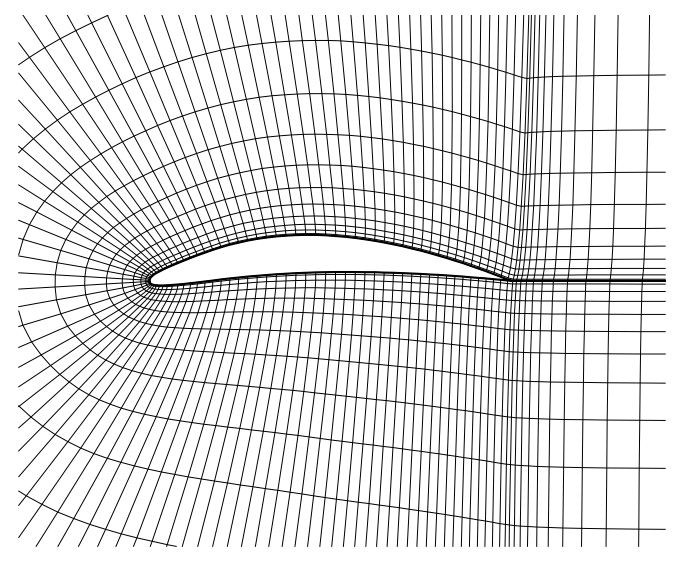

Figure 3: Sample C-type grid

(elliptic, parabolic, etc.) as desired for different flight regions. In this manner, the number of design parameters can be kept to a minimum, which is an important factor in reducing the optimization costs.

The algebraic grid generation system, used in this study, is an explicit mathematical expression of a physical domain as a function of a computational domain. A methodology based on separating the boundary definition from the interior definition is established. The interior is then defined as a function of information on the boundaries such as position, surface derivatives, and an independent variable. An example of such formulation with first-order surface derivatives is called Two-Boundary Grid Generation (TBGG) technique. ${ }^{7}$ This, matches both the function and its derivative at the boundaries. Figure 3 illustrating the resultant sample grid for the airfoil geometry using this technique.

\section{FLOW ANALYSIS AND SENSITIVITY EQUATION}

\subsection{Analysis}

The two-dimensional thin-layer Navier-Stokes equations can be represented as

$$
\frac{1}{J} \frac{\partial \mathbf{Q}}{\partial t}=\mathbf{R}(\mathbf{Q}) \quad \mathbf{Q}=\left\{\begin{array}{c}
\rho \\
\rho u \\
\rho v \\
e
\end{array}\right\}_{i, j} .
$$

Here, $\mathbf{R}$ is the residual and $J$ is the transformation
Jacobian

$$
J=\frac{\partial(\xi, \eta)}{\partial(x, y)} .
$$

The residual $\mathbf{R}$ can be expressed in generalized curvilinear coordinates $(\xi, \eta)$ as,

$$
\mathbf{R}=-\frac{\partial \overline{\mathbf{F}}}{\partial \xi}-\frac{\partial\left(\overline{\mathbf{G}}-\overline{\mathbf{G}}_{\mathbf{V}}\right)}{\partial \eta}
$$

where $\overline{\mathbf{F}}$ and $\overline{\mathbf{G}}$ are the inviscid and $\overline{\mathbf{G}}_{\mathbf{v}}$ is the viscous fluxes.

The equations are solved in their conservation form using an upwind cell-centered finite-volume formulation. A third-order accurate upwind biased inviscid flux balance is used in both streamwise and normal directions. The finite-volume equivalent of secondorder accurate central differences is used for viscous terms. The resulting discretization represents the residual, $\mathbf{R}(\mathbf{Q})$, at each cell depending locally on values of $\mathbf{Q}$ at nine neighboring cells such that

$$
\begin{aligned}
\mathbf{R}_{i, j}(\mathbf{Q})= & \mathbf{R}_{i, j}\left(\mathbf{Q}_{i, j}, \mathbf{Q}_{i, j-1}, \mathbf{Q}_{i, j+1}, \mathbf{Q}_{i, j-2}, \mathbf{Q}_{i, j+2},\right. \\
& \left.\mathbf{Q}_{i-1, j}, \mathbf{Q}_{i+1, j}, \mathbf{Q}_{i-2, j}, \mathbf{Q}_{i+2, j}\right) .
\end{aligned}
$$

The discretized governing equations are implicitly advanced in time using Euler implicit method which is unconditionally stable for all time steps according to Fourier stability analysis. An iterative approximate factorization (AF) algorithm have been chosen to advance the solution in time until

$$
\mathbf{R}\left(\mathbf{Q}^{*}\right) \approx 0
$$

where $\mathbf{Q}^{*}$ are the steady-state values of the field variables. The boundary conditions are implicitly implemented within the governing equations. The airfoils surface is considered to be impermeable and adiabatic. A standard no-slip boundary condition with zero surface velocity has been selected. The pressure at the surface is evaluated using a zeroth-order extrapolation from the interior cells. The density is then calculated using the state equation.

\subsection{Sensitivity}

For a steady-state solution (i.e., $t \rightarrow \infty$ ), Eq.(6) is reduces to

$$
\mathbf{R}\left(\mathbf{Q}^{*}(\mathbf{P}), \mathbf{X}(\mathbf{P}), \mathbf{P}\right)=0
$$

where the explicit dependency of $\mathbf{R}$ on grid and vector of parameters $\mathbf{P}$ is evident. The parameters $\mathbf{P}$ control the grid $\mathbf{X}$ as well as the solution $\mathbf{Q}^{*}$. The fundamental sensitivity equation containing $\left\{\frac{\partial \mathbf{Q}^{*}}{\partial \mathbf{P}}\right\}$ 
and described by Taylor et al. ${ }^{2}$ is obtained by direct differentiation of Eq.(7) as

$$
\left[\frac{\partial \mathbf{R}}{\partial \mathbf{Q}}\right]\left\{\frac{\partial \mathbf{Q}^{*}}{\partial \mathbf{P}}\right\}+\left[\frac{\partial \mathbf{R}}{\partial \mathbf{X}}\right]\left\{\frac{\partial \mathbf{X}}{\partial \mathbf{P}}\right\}=0 .
$$

It is important to notice that Eq.(8) is a set of linear algebraic equations, and the matrices $\left[\frac{\partial \mathbf{R}}{\partial \mathbf{Q}}\right]$ and $\left[\frac{\partial \mathbf{R}}{\partial \mathbf{X}}\right]$ are well understood. The vector quantity $\left\{\frac{\partial \mathbf{Q}^{*}}{\partial \mathbf{P}}\right\}$ is the solution to Eq.(8) given the sensitivity of the grid with respect to the parameters, $\left\{\frac{\partial \mathbf{X}}{\partial \mathbf{P}}\right\}$. A direct chain rule differentiation of $\left\{\frac{\partial \mathbf{X}}{\partial \mathbf{P}}\right\}$ results in

$$
\left\{\frac{\partial \mathbf{X}}{\partial \mathbf{P}}\right\}=\left[\frac{\partial \mathbf{X}}{\partial \mathbf{X}_{B}}\right]\left\{\frac{\partial \mathbf{X}_{B}}{\partial \mathbf{P}}\right\}
$$

where $\mathbf{X}_{B}$ designates the boundary coordinates. The vector $\left\{\frac{\partial \mathbf{X}_{B}}{\partial \mathbf{P}}\right\}$ represents the boundary sensitivity which is directly related to boundary parameterization, discussed previously. It has the importance of being one of the dominant factors in calculating the sensitivity of surface forces needed for optimization process. The matrix $\left[\frac{\partial \mathbf{X}}{\partial \mathbf{X}_{B}}\right]$ is responsible for field grid sensitivity with respect to boundary coordinates and it is related to the rules which govern the grid generation algorithm. For algebraic generation systems, the primary components of $\left[\frac{\partial \mathbf{X}}{\partial \mathbf{X}_{B}}\right]$, are the interpolation functions which distribute the interior grid.

The sensitivity of the grid with respect to the vector of design parameters $\mathbf{X}_{D}=\left\{X_{i}, Y_{i}, \omega_{i}\right\}^{T}$ can be obtained by direct differentiation of the grid equations. ${ }^{5}$ As a consequence of using algebraic grid generation technique in which the boundary grid has the dominant effect on the interior grid, the boundary grid sensitivity coefficient would also be essential in influencing the interior grid sensitivity coefficient. Therefore, evaluation of the surface grid sensitivity coefficients are the most important part of the analysis and are directly dependent on the surface parameterization. For practical purposes, the grid sensitivity and orthogonality at the far-field boundary has been ignored.

The flow sensitivity coefficient $\left\{\frac{\partial \mathbf{Q}^{*}}{\partial \mathbf{P}}\right\}$ can now be directly obtained using the fundamental sensitivity equation, Eq.(8), as

$$
\left\{\frac{\partial \mathbf{Q}^{*}}{\partial \mathbf{P}}\right\}=-\left[\frac{\partial \mathbf{R}}{\partial \mathbf{Q}}\right]^{-1}\left[\frac{\partial \mathbf{R}}{\partial \mathbf{X}}\right]\left\{\frac{\partial \mathbf{X}}{\partial \mathbf{P}}\right\}
$$

provided that grid sensitivity, $\left\{\frac{\partial \mathbf{X}}{\partial \mathbf{P}}\right\}$, is known. The Jacobian matrix, $\left[\frac{\partial \mathbf{R}}{\partial \mathbf{X}}\right]$, can be evaluated by differentiating the discrete residuals $\mathbf{R}_{i, j}$ with respect to four vertices of each cell. The quantity $\left[\frac{\partial \mathbf{R}}{\partial \mathbf{Q}}\right]^{-1}$ can be obtained using a full matrix solver to account for all the non-zero contributions outside of central bandwidth. This, although convenient, is not practical for Navier-Stokes equations due to large storage requirements. An alternative would be the use of a hybrid direct solver with conventional relaxation strategy. ${ }^{2}$

\subsection{Optimization}

An objective of a multidisciplinary optimization of a vehicle design is to extremize a payoff function combining dependent parameters from several disciplines. Most optimization techniques require the sensitivity of the payoff function with respect to free parameters of the system. For a fixed grid and solution conditions, the only free parameters are the surface design parameters. Therefore, the sensitivity of the payoff function with respect to design parameters is needed. The optimization problem is based on the method of feasible directions and the generalized reduced gradient method. ${ }^{8}$ This method has the advantage of progressing rapidly to a near-optimum design with only gradient information of the objective and constrained functions required. The problem can be defined as finding the vector of design parameters $\mathbf{X}_{D}$, which will minimize the objective function $f\left(\mathbf{X}_{D}\right)$ subjected to constraints

$$
g_{j}\left(\mathbf{X}_{D}\right) \leq 0 \quad j=1, m
$$

and

$$
\mathbf{X}_{D}^{l} \leq \mathbf{X}_{D} \leq \mathbf{X}_{D}^{u}
$$

where superscripts denote the upper and lower bounds for each design parameter. The optimization process proceeds iteratively as

$$
\mathbf{X}_{D}^{n}=\mathbf{X}_{D}^{n-1}+\gamma \overline{\mathbf{S}}^{n}
$$

where $n$ is the iteration number, $\overline{\mathbf{S}}^{n}$ the vector of search direction, and $\gamma$ a scalar move parameter. The first step is to determine a feasible search direction $\overline{\mathbf{S}}^{n}$, and then perform a one-dimensional search in this direction to reduce the objective function as much as possible, subjected to the constraints.

The present optimization strategy is based on maximizing the lift coefficient, $C_{L}$, in response to surface perturbation, subject to pre-determined design constraints. Upper and lower bounds set for each design parameter and the sensitivity derivatives of the objective function, $\frac{\partial C_{L}}{\partial \mathbf{X}_{D}}$, and the constraint, $\frac{\partial C_{D}}{\partial \mathbf{X}_{D}}$, 


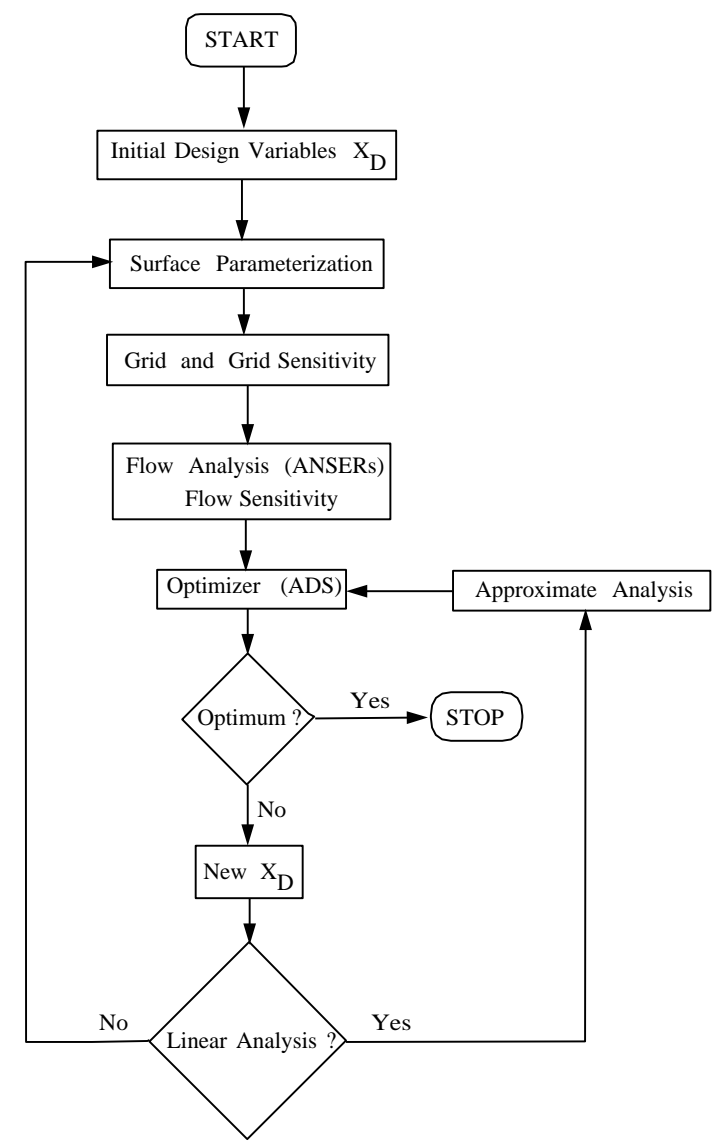

Figure 4: Optimization strategy loop

are obtained as previously described. ${ }^{2,3}$ Throughout the analysis, the drag coefficient, $C_{D}$, is to be no greater than the value of the initial design. The strategy, illustrated in Fig. 4, requires that the grid and grid sensitivity derivatives be provided dynamically during the automated optimization process.

\section{RESULTS AND DISCUSSION}

\subsection{Grid Sensitivity}

The grid sensitivity of a generic airfoil with respect to design parameters using the NURBS parameterization is discussed in this section. The geometry, as shown in Fig. 1, has seven pre-specified control points. The control points are numbered counterclockwise, starting and ending with control points ( 0 and 6), assigned to the tail of the airfoil. A total of 21 design parameters (i.e., three design parameters per control point) available for optimization purpose. Depending on desired accuracy and degree of freedom for optimization, the number of design parameters could be reduced for each particular problem. For

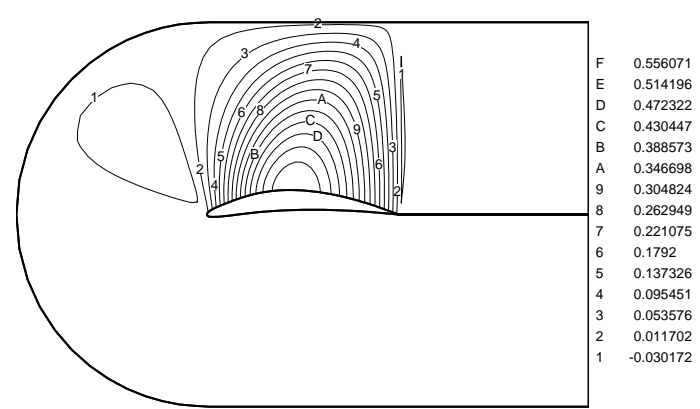

(a) Y-Coordinate

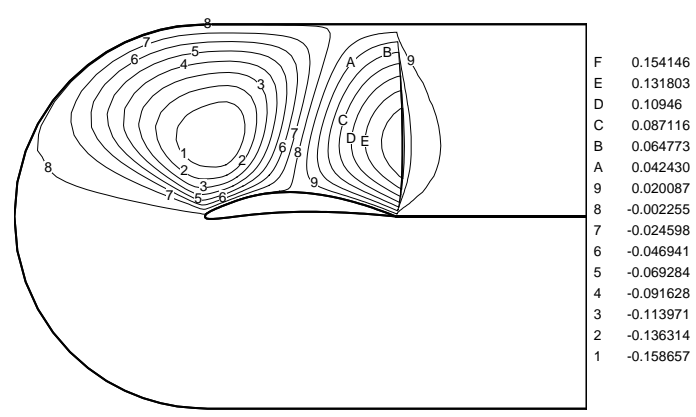

(b) X-Coordinate

Figure 5: Grid sensitivity with respect to $Y_{1}$

the present case, such reduction is achieved by considering fixed weights and chord-length. Out of the remaining four control points with two degrees of freedom for each, control points 1 and 5 have been chosen as a case study. The number of design parameters is now reduced to four with $\mathbf{X}_{D}=\left\{X_{1}, Y_{1}, X_{5}, Y_{5}\right\}^{T}$, with initial values specified in Fig.1. The non-zero contribution to the surface grid sensitivity coefficients of these control points are the basis functions $R_{1,3}(r)$ and $R_{5,3}(r)$. Figure 5 illustrates the field-grid sensitivity with respect to design parameter $Y_{1}$ when the far-field boundary is placed one chord-length away from the surface. The sensitivity gradients are restricted only to the region influenced by the elected control point. This locality feature of the NURBS parameterization makes it a desirable tool for complex design and optimization when only a local perturbation of the geometry is warranted. Similar results can be obtained for design control point 5 where the sensitivity gradients are restricted to the lower portion of domain. 


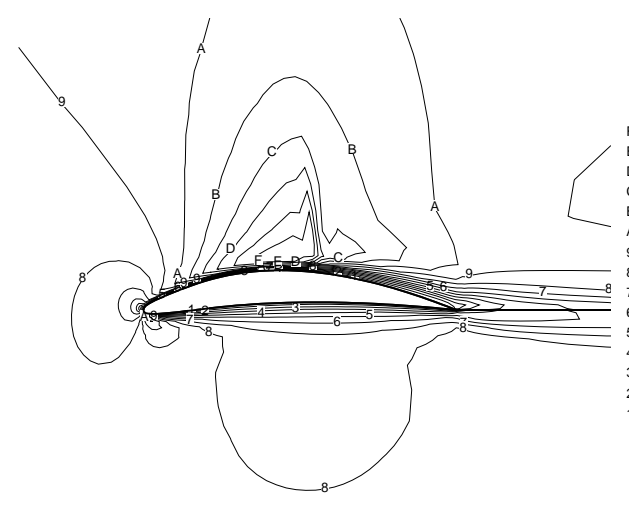

Figure 6: Mach number contours $\left(\alpha=0, M_{\infty}=0.7\right)$

\subsection{Flow Sensitivity and Optimization}

The second phase of the problem is obtaining the flow sensitivity coefficients using the previously obtained grid sensitivity coefficients. In order to achieve this, according to Eq.(8), a converged flow field solution about a fixed design point should be obtained. The computation is performed on a C-type grid composed of 141 points in the streamwise direction with 101 points on the airfoil surface, and 31 points in the normal direction. The far-field and outer boundary were placed about 20 chord-length away from the airfoils. It is apparent that such a coarse grid is inadequate for capturing the full physics of the viscous flow over an airfoils. Therefore, it should be understood that the main objective here is not to produce a highly accurate flow field solution rather than to demonstrate the feasibility of the approach.

The two-dimensional, compressible, thin-layer Navier-Stokes equations are solved for a free stream Mach number of $M_{\infty}=0.7$, Reynolds number $R e_{\infty}=10^{6}$, and angle of attack $\alpha=0^{\circ}$. The solution is implicitly advanced in time using local time stepping as a means of promoting convergence toward the steady-state. The residual is reduced by ten orders of magnitude. All computations are performed on NASA Langley's Cray-2 mainframe with a computation cost of $0.1209 \times 10^{-3} \mathrm{CPU}$ seconds/iteration/grid point. Figure 6 demonstrates the Mach number contours of the converged solution with the lift and drag coefficients of $C_{L}=0.402$ and $C_{D}=0.063$. Due to surface curvature, the flow accelerates along the the upper surface to supersonic speeds, terminated by a weak shock wave behind which it becomes subsonic. The sensitivity coefficient, $\left\{\frac{\partial \mathbf{Q}^{*}}{\partial T}\right\}$, is obtained

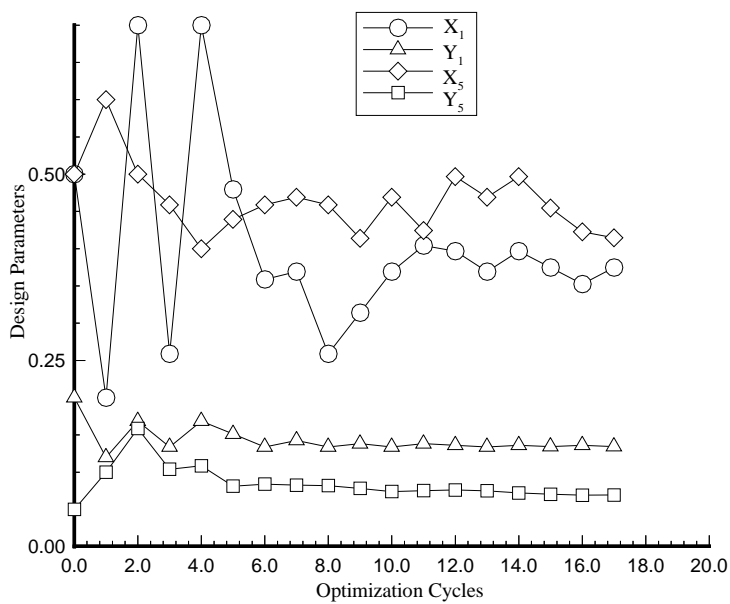

Figure 7: Optimization cycles history

by previously described iterative strategy. ${ }^{2}$ The average relative error has been reduced by three orders of magnitude. The sensitivities of the aerodynamic forces, such as drag and lift coefficients with respect to design parameters $\left\{X_{1}, Y_{1}, X_{5}, Y_{5}\right\}^{T}$, are obtained and results are presented in Table 1 . An inspection of Table 1 indicates the substantial influence of parameters $Y_{1}$ and $Y_{5}$ on the aerodynamic forces acting on the surface. The upper and lower bounds for these design parameters are assigned as

$$
\begin{array}{ll}
0.2 \leq X_{1} \leq 0.7, & -0.1 \leq Y_{1} \leq 0.5, \\
0.2 \leq X_{5} \leq 0.7, & -0.1 \leq Y_{5} \leq 0.2 .
\end{array}
$$

The optimum design is achieved after 17 optimization cycles and a total of 8807 Cray-2 CPU seconds. These high computational costs make minimizing the number of design parameters in optimization cycle essential. Table 2 highlights the initial and final values of lift and drag coefficients with a $208 \%$ improvement in their ratio. Table 3 represents the initial and optimum design parameters with parameters $Y_{1}$ and $Y_{5}$ having the largest change as expected. The history of design parameters deformation during the optimization cycles appears in Fig. 7, where the oscillatory nature of design perturbations during the early cycles are clearly visible. Figure 8 compares the original and optimum geometry of the airfoil.

Several observations should be made at this point. First, although control points 1 and 5 demonstrated to have substantial influence on the design of the airfoil, they are not the only control points affecting the design. In fact, control points 2 and 4 near the nose might have greater affect due to sensitive nature of lift and drag forces on this region. The choice 


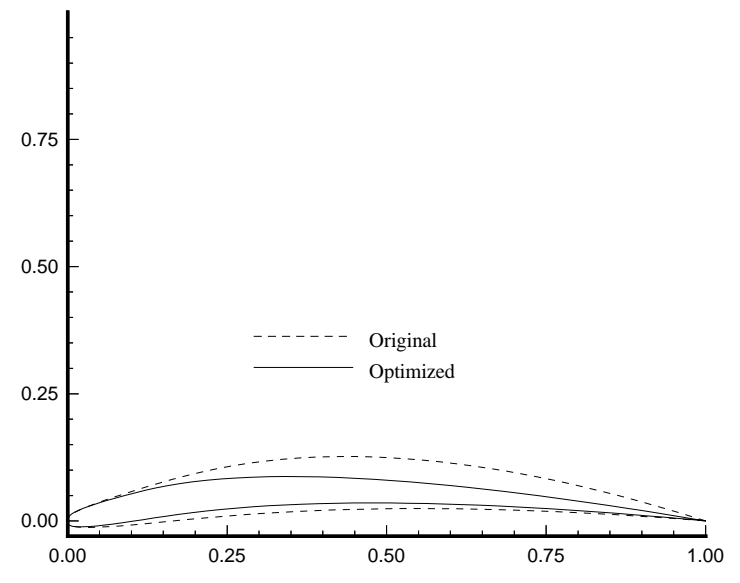

Figure 8: Original and optimized airfoils

of control points 1 and 5 here was largely based on their camber like behavior. A complete design and optimization should include all the relevant control points (e.g., control points 1, 2, 4, and 5). For geometries with large number of control points, in order to contain the computational costs within a reasonable range, a criteria for selecting the most influential control points for optimization purposes should be established. This decision could be based on the already known sensitivity coefficients, where control points having the largest coefficients could be chosen as design parameters. Secondly, the optimum airfoil of Fig. 8 is only valid for this particular example and design range. As a direct consequence of the nonlinear nature of governing equations and their sensitivity coefficients, the validity of this optimum design would be restricted to a very small range of the original design parameters. The best estimate for this range would be the finite-difference step size used to confirm the sensitivity coefficients (i.e., $10^{-3}$ or less). All the airfoils with the original control points within this range should conform to the optimum design of Fig. 8, while keeping the grid and flow conditions fixed.

Table 1 Aerodynamic sensitivity coefficients

\begin{tabular}{|l|l|r|}
\hline \hline Generic Wing-Section & \multicolumn{2}{|c|}{ Direct Differentiation } \\
\hline Design Parameters: & $\frac{\partial C_{L}}{\partial X_{D}}$ & $\frac{\partial C_{D}}{\partial X_{D}}$ \\
\hline$X_{1}$ & -0.297 & $-3.63 \times 10^{-2}$ \\
\hline$Y_{1}$ & -5.107 & 0.549 \\
\hline$X_{5}$ & 0.15 & $-2.04 \times 10^{-2}$ \\
\hline$Y_{5}$ & 2.609 & 0.287 \\
\hline \hline
\end{tabular}

Table 2 Comparison of initial and optimized performance variables

\begin{tabular}{|l|l|l|r|}
\hline \hline $\begin{array}{l}\text { Performance } \\
\text { Variables }\end{array}$ & $\begin{array}{l}\text { Initial } \\
\text { Design }\end{array}$ & $\begin{array}{l}\text { Optimum } \\
\text { Design }\end{array}$ & $\begin{array}{r}\text { Percent } \\
\text { Change }\end{array}$ \\
\hline$C_{L}$ & 0.402 & 0.845 & +110.1 \\
\hline$C_{D}$ & 0.063 & 0.043 & -31.7 \\
\hline Lift/Drag Ratio & 6.38 & 19.65 & +208 \\
\hline \hline
\end{tabular}

Table 3 Comparison of initial and optimized design parameters

\begin{tabular}{|l|l|l|r|}
\hline \hline $\begin{array}{l}\text { Design } \\
\text { Parameters }\end{array}$ & $\begin{array}{l}\text { Initial } \\
\text { Design }\end{array}$ & $\begin{array}{l}\text { Optimum } \\
\text { Design }\end{array}$ & $\begin{array}{r}\text { Percent } \\
\text { Change }\end{array}$ \\
\hline$X_{1}$ & 0.5 & 0.374 & -25.2 \\
\hline$Y_{1}$ & 0.2 & 0.134 & -33 \\
\hline$X_{5}$ & 0.5 & 0.414 & -17.2 \\
\hline$Y_{5}$ & 0.05 & 0.069 & +38 \\
\hline \hline
\end{tabular}

\section{CONCLUSIONS AND RECOMMENDATIONS}

An algorithm is developed to obtain the grid sensitivity with respect to design parameters for aerodynamic optimization. The algebraic Two-Boundary Grid Generation (TBGG) scheme has been directly differentiated with respect to design parameters. This formulation has the benefits of being exact, efficient, and inexpensive. The airfoil is defined geometrically using the NURBS approximation of the surface. A substantial increase in aerodynamic performance variables enforces the feasibility of this approach for high level design and optimization.

It is evident that grid sensitivity plays a significant role in the aerodynamic optimization process. The algebraic grid generation scheme presented here is intended to demonstrate the elements involved in obtaining the grid sensitivity from an algebraic grid generation system. Each grid generation formulation requires considerable analytical differentiation with respect to parameters which control the boundaries as well as the interior grid. It is implied that aerodynamic surfaces, such as the airfoil considered here, should be parameterized in terms of design parameters. Due to the high cost of aerodynamic optimization process, it is imperative to keep the number of design parameters as low as possible. Analytical parameterization, although facilitates this notion, has the disadvantage of being restricted to simple geometries. A geometric parameterization such as NURBS, with local sensitivity, has been advocated for more complex geometries. 
Future investigations should include the implementation of present approach using larger grid dimensions, adequate to resolve full physics of viscous flow analysis. A grid optimization mechanism based on grid sensitivity coefficients with respect to grid parameters should be included in the overall optimization process. An optimized grid applied to present geometry, should increase the quality and convergence rate of flow analysis within optimization cycles. Other directions could be establishing a link between geometric design parameters (e.g., control points and weights) and basic physical design parameters (e.g., camber and thickness). This would provide a consistent model throughout the analysis which could easily be modified for optimization. Also, the effects of including all the relevant control points on the design cycles should be investigated. Another contribution would be the extension of the current algorithm to three-dimensional space for complex applications. For three-dimensional applications, even a geometric parameterization of a complete aerodynamic surface can require a large number of parameters for its definition. A hybrid approach can be selected when certain sections or skeleton parts of a surface are specified with NURBS and interpolation formulas are used for intermediate surfaces.

\section{ACKNOWLEDGEMENTS}

The authors would like to thank Drs. Arthur C. Taylor III and Oktay Baysal of Old Dominion University for their helpful suggestions and guidance throughout this investigation. This work was supported by the NASA Langley Research Center through Cooperate Agreement NCC1-68.

\section{REFERENCES}

${ }^{1}$ Sobieszczanski-Sobieski, J. "Sensitivity Analysis and Multidisciplinary Optimization for Aircraft Design : Recent Advances and Results," NASA TM100630, July 1988.

${ }^{2}$ Taylor, A. C., III, Hou, G. W., and Korivi, V. M., "Sensitivity Analysis, and Design Optimization For Internal and External Viscous Flows," AIAA Paper 91-3083, September 23-25, 1991, Baltimore, MD.

${ }^{3}$ Baysal, O., and Eleshaky, M. E., "Aerodynamic Design Optimization Using Sensitivity Analysis and Computational Fluid Dynamics," AIAA Paper 910471, January 1991. Also appeared in AIA A Journal, Volume 30, Number 3, March 1992, pp.718-725.

${ }^{4}$ Sadrehaghighi, I., Smith, R.E., Tiwari, S.N.,
"Grid and Aerodynamic Sensitivity Analyses of Airplain Components," AIAA Paper 93-3475, $11^{\text {th }}$ Applied Aerodynamic Conference, Monterey, CA, August 9-11, 1993.

${ }^{5}$ Sadrehaghighi, I., Smith, R.E., Tiwari, S.N., "Grid and Design Variables Sensitivity Analyses for NACA Four-Digit Wing-Sections," AIAA Paper 930195, 30 ${ }^{\text {th }}$ Aerospace Sciences Meeting, Reno, NV, January 11-14, 1993.

${ }^{6}$ Tiller, W., "Rational B-Splines for Curve and Surface Representation," Computer Graphics and Applications, Volume 3, N0. 10, September 1983, pp. 6169.

${ }^{7}$ Smith, R. E., and Wiese, M. R., "Interactive Algebraic Grid-Generation Technique," NASA Technical Paper 2533, March 1986.

${ }^{8}$ Vanderplaats, G.N., " ADS - A Fortran Program for Authomated Design Synthesis," NASA CR177985, September 1985. 